\title{
Anti-breast cancer activity of some novel quinoline derivatives
}

MOSTAFA M. GHORAB*

MANSOUR S. ALSAID

Department of Pharmacognosy

College of Pharmacy

King Saud University

P.O. Box 2457, Riyadh 11451

Saudi Arabia

Accepted July 1, 2015
To discover new bioactive lead compounds for medicinal purposes, 2-cyano-3-(4-substituted)- $N$-(quinolin-3-yl) acrylamide derivatives 2-24, chromenes 25, 26 and benzochromenes $\mathbf{2 7}, \mathbf{2 8}$ were synthesized. The structures of the newly synthesized compounds were confirmed by elemental analyses, IR, ${ }^{1} \mathrm{H}$ NMR and ${ }^{13} \mathrm{C}$ NMR spectroscopies. In addition, the structure of compound $\mathbf{1}$ was confirmed through X-ray crystallography. All the newly synthesized compounds were evaluated for their cytotoxic activity against the breast cancer cell line MCF7. The corresponding 2-cyano-3-(4-hydroxy-3-methoxyphenyl)- $N$-(quinolin-3-yl) acrylamide (15), 3-oxo- $N$-(quinolin-3-yl)-3H-benzol[ $f]$ chromene-2-carboxamide (27), 2-cyano-3-(4-fluorophenyl$N$-(quinolin-3-yl) acrylamide (7), 2-cyano-5-(4-(dimethylamino) phenyl)- $N$-(quinolin-3-yl) penta-2,4-dienamide (19) exhibited higher activity compared to doxorubicin (with $I C_{50}$ value of $\left.47.9 \mu \mathrm{mol} \mathrm{L}-1\right)$ as a reference drug, with $I C_{50}$ values of $29.8,39.0,40.0,40.4 \mu \mathrm{mol} \mathrm{L}^{-1}$, resp. Also, quinoline acrylamides containing 2,3,4-trimethoxyphenyl 17, 2-chlorophenyl 10, benzo[d][1,3]dioxol 12, 2-methoxynaphthalen 22, 2,4-dichlorophenyl 18 and quinoline carrying a chromene-3-carboxamide moiety $\mathbf{2 5}$ were nearly as active as doxorubicin, while quinoline acrylamides incorporating unsubstituted phenyl 2, p-tolyl 3, 2,4-dienamide 8, 3-nitrophenyl 13, 4-nitrophenyl 14, 3,4-dimethoxyphenyl 16 and chromene 26 exhibited a moderate activity. In addition, quinoline with acetamide 1, 4-hydroxyphenyl 4, 4-dimethylaminophenyl 9, 4-chlorophenyl 11, 3-bromophenyl 20, 4-bromophenyl 21 and 3-thienyl moiety 24 showed less activity than doxorubicin. On the other hand, quinoline having 2-methoxyphenyl 5, 4-methoxyphenyl 6, 4-methoxynaphthalene $\mathbf{2 3}$ and chromene-2-carboxamide $\mathbf{2 8}$ showed no activity.

Keywords: quinolineacrylamides, chromenes, benzochromenes, anti-breast cancer activity

\footnotetext{
*Correspondence; e-mail: mmsghorab@yahoo.com
} 
Several quinoline derivatives, isolated from natural sources or prepared synthetically, are important for medicinal chemistry and biomedical use. The bark of Cinchona plant containing quinine has been utilized to treat palpitations (1), fevers and tertians for more than 200 years. Quinidine, a diastereo isomer of quinine, was regarded in the early $20^{\text {th }}$ century as the most potent antiarrhythmic compound isolated from the Cinchona plant (2). The quinoline skeleton is often used to design many synthetic compounds with diverse pharmacological properties, such as anti-inflammatory (3), antimicrobial (4), cytotoxic (5), antibacterial (6) and antitumor activity (7). In addition, quinoline derivatives find use in the synthesis of fungicides, virucides, biocides, alkaloids and flavoring agents $(8,9)$. Quinolines also act as carbonic anhydrase inhibitors $(10,11)$. On the other hand, various fused systems of quinolines were studied for their intercalative DNA binding properties. A literature survey reveals that the antitumor activity is due to the intercalation between the base pairs of DNA and interferences with the normal functioning of enzyme topoisomerase II, which is involved in the breaking and releasing of DNA strands (12). The antitumor drugs that intercalate DNA are of growing interest in the field of anticancer derivatives. On the other hand, doxorubicin, the reference drug used in this study, is a drug used in cancer chemotherapy. It is an anthracycline antibiotic and works by intercalating DNA and inhibiting of macromolecular biosynthesis. This inhibits the progression of the enzyme topoisomerase II, which relaxes super coils in DNA for transcription. Doxorubicin stabilizes the topoisomerase II complex after it has broken the DNA chain for replication, preventing the DNA double helix from being resealed and thereby stopping the process of replication. It is commonly used in the treatment of a wide range of cancers such as acute leukemia Hodgkin's disease and other lymphomas and cancers of the breast, adrenal cortex, endometrial, lung, ovary, and other sites. As a part of our ongoing research program directed towards developing new approaches to a variety of heterocyclic ring systems for anticancer activity, especially those containing nitrogen compounds (13-21), we herein report the utility of strategic starting material 3-aminoquinoline for the synthesis of target compounds.

\section{EXPERIMENTAL}

Melting points (uncorrected) were determined in an open capillary in a Gallenkamp melting point apparatus (Sanyo Gallenkamp, UK). Precoated silica gel plates (Kieselgel $0.25 \mathrm{~mm}, 60 \mathrm{~F} 254$, Merck, Germany) were used for thin layer chromatography. A developing solvent system of chloroform/methanol (8:2) was used and the spots were detected by ultraviolet light. IR spectra ( $\mathrm{KBr}$ disc) were recorded using an FT-IR spectrophotometer (Perkin Elmer, USA). ${ }^{1} \mathrm{H}$ NMR spectra were scanned on a NMR spectrophotometer (Bruker AXS Inc., Switzerland), operating at $500 \mathrm{MHz}$ for ${ }^{1} \mathrm{H}$ - and $125.76 \mathrm{MHz}$ for ${ }^{13} \mathrm{C} \mathrm{NMR}$. Chemical shifts are expressed in $\delta$ values (ppm) relative to TMS as an internal standard, using DMSO- $d_{6}$ as a solvent. Elemental analyses were done on a model 2400 CHNSO analyser (Perkin Elmer, USA). All values were within $\pm 0.4 \%$ of the theoretical values. All reagents used were of AR grade. The starting material 3-aminoquinoline was purchased from Sigma (USA) and was directly used for the preparation of target compounds.

\section{Syntheses}

2-Cyano-N-(quinolin-3-yl) acetamide (1). - A mixture of 3-aminoquinoline (1.44 g, 0.01 mol) and ethyl cyanoacetate $(1.13 \mathrm{~g}, 0.01 \mathrm{~mol})$ was fused at $220{ }^{\circ} \mathrm{C}$ for $2 \mathrm{~h}$. The reaction 
mixture was cooled and the obtained product was crystallized from ethanol to give $\mathbf{1}$ (Table I).

General procedure for 2-cyano-3-phenyl-N-(quinolin-3-yl) acrylamide (2), 2-cyano-N-(quinolin-3-yl)-3-p-tolylacrylamide (3), cyano-3-(4-hydroxyphenyl)-N-(quinolin-3-yl) acrylamide (4), 2-cyano-3-(2-methoxyphenyl)-N-(quinolin-3-yl) acrylamide (5), 2-cyano-3-(4-methoxyphenyl)-N(quinolin-3-yl) acrylamide (6), 2-cyano-3-(4-fluorophenyl-N-(quinolin-3-yl) acrylamide (7), 2-cyano-5-phenyl- N-(quinolin-3-yl) penta-2,4-dienamide (8), 2-cyano-3-(4-(dimethylamino) phenyl)$\mathrm{N}$-(quinolin-3-yl) acrylamide (9), 3-(2-chlorophenyl)-2-cyano-N-(quinolin-3-yl) acrylamide (10), 3-(4-chlorophenyl)-2-cyano-N-(quinolin-3-yl) acrylamide (11), 3-(benzo[d] [1,3] dioxol-5-yl)-2cyano-N-(quinolin-3-yl) acrylamide (12), 2-cyano-3-(3-nitrophenyl)-N-(quinolin-3-yl) acrylamide (13), 2-cyano-3-(4-nitrophenyl)-N-(quinolin-3-yl) acrylamide (14), 2-cyano-3-(4-hydroxy-3-methoxyphenyl)-N-(quinolin-3-yl) acrylamide (15), 2-cyano-3-(3,4-dimethoxyphenyl)-N-(quinolin-3yl) acrylamide (16), 2-cyano-N-(quinolin-3-yl)-3-(2,3,4-trimethoxyphenyl) acrylamide (17), 2-cyano-3-(2,4-dichorophenyl)-N-(quinolin-3-yl) acrylamide (18), 2-cyano-5-(4-(dimethylamino) phenyl)-N-(quinolin-3-yl) penta-2,4-dienamide (19), 3-(3-bromophenyl)-2-cyano-N-(quinolin-3yl) acrylamide (20), 3-(4-bromophenyl)-2-cyano-N-(quinolin-3-yl) acrylamide (21), 2-cyano-3-(2methoxynaphthalen-1-yl)-N-(quinolin-3-yl) acrylamide (22), 2-cyano-3-(4-methoxynapthalen-1yl)-N-(quinolin-3-yl) acrylamide (23), 2-cyano-N-(quinolin-3-yl)-3-(thiophen-2-yl) acrylamide (24) - A mixture of 1 ( $2.11 \mathrm{~g}, 0.01 \mathrm{~mol})$ and appropriate aldehyde $(0.01 \mathrm{~mol})$ in absolute ethanol $(20 \mathrm{~mL}$ ) containing 3 drops of piperidine was refluxed for $8 \mathrm{~h}$. The reaction mixture was cooled and poured into ice/water. The solid obtained was recrystallized from dioxane to give quinoline derivatives 2-24, respectively (Table I).

General procedure for 2-oxo-N-(quinolin-3-yl)-2H-chromene-3-carboxamide (25) and 3-oxo$\mathrm{N}$-(quinolin-3-yl)-3H-benzol[f]chromene-2-carboxamide (27) - To a solution of 1 (2.11 g, 0.01 $\mathrm{mol})$ in acetic anhydride $(20 \mathrm{~mL})$, salicylaldehyde $(1.22 \mathrm{~g}, 0.01 \mathrm{~mol})$ or 2-hydroxy-1-naphthaldehyde $(1.72 \mathrm{~g}, 0.01 \mathrm{~mol})$ and fused sodium acetate $(0.8 \mathrm{~g}, 0.01 \mathrm{~mol})$ were added. The reaction mixture was refluxed for $2 \mathrm{~h}$, cooled and the solid was filtered and crystallized from ethanol to give 25 and 27, respectively (Table I).

General procedure for 2-imino-N-(quinolin-3-yl)-2H-chromene-3-carboxamide (26) and 3-imino-N-(quinolin-3-yl)-3H-benzol[f]chromene-2-carboxamide (28) - A mixture of compound 1 ( $2.11 \mathrm{~g}, 0.01 \mathrm{~mol})$, salicylaldehyde $(1.22 \mathrm{~g}, 0.01 \mathrm{~mol})$ or 2-hydroxy-1-naphthyladehyde (1.72 $\mathrm{g}, 0.01 \mathrm{~mol})$ and anhydrous ammonium acetate $(1.15 \mathrm{~g}, 0.015 \mathrm{~mol})$ was refluxed in absolute ethanol $(30 \mathrm{~mL})$ for $2 \mathrm{~h}$. The obtained solid was recrystallized from ethanol to give 27 and 29, respectively (Table I).

\section{In vitro anti-breast cancer activity}

In vitro cytotoxic activity of the synthesized compounds was measured using the sulforhodamine B stain (SRB) assay after the method of Skehan (22). The human breast cancer cell line MCF7 was obtained from the National Cancer Institute (Cairo, Egypt). The cell lines were grown in RPMI 1640 medium containing $10 \%$ fetal bovine serum (Lonza, Switzerland), $100 \mathrm{IU} \mathrm{mL} \mathrm{m}^{-1}$ penicillin, $100 \mathrm{mg} \mathrm{mL}^{-1}$ streptomycin and $2 \mathrm{mmol} \mathrm{L}^{-1}$ L-glutamine (Sigma, USA). Cells were plated in 96-multiwell plates ( $10^{4}$ cells/well). After cell inoculation, the micro titer plates were incubated at $37{ }^{\circ} \mathrm{C}$ in $5 \% \mathrm{CO}_{2}, 95 \%$ air and $100 \%$ relative humidity for $24 \mathrm{~h}$ prior to addition of experimental drugs to allow attachment of cells to the plate wall. After $24 \mathrm{~h}$, cell line was fixed in situ with TCA (trichloroacetic acid). 
Table I. Physical and analytical data of the newly synthesized compounds

\begin{tabular}{|c|c|c|c|c|c|c|}
\hline \multirow{2}{*}{$\begin{array}{c}\text { Compd. } \\
\text { No. }\end{array}$} & \multirow{2}{*}{$\begin{array}{l}\text { Formula } \\
\left(M_{\mathrm{r}}\right)\end{array}$} & \multirow{2}{*}{$\begin{array}{l}\text { M. p. } \\
\left({ }^{\circ} \mathrm{C}\right)\end{array}$} & \multirow{2}{*}{$\begin{array}{l}\text { Yield } \\
(\%)\end{array}$} & \multicolumn{3}{|c|}{ Analysis (calcd/found) (\%) } \\
\hline & & & & C & $\mathrm{H}$ & $\mathrm{N}$ \\
\hline 1 & $\mathrm{C}_{12} \mathrm{H}_{9} \mathrm{~N}_{3} \mathrm{O}(211.22)$ & 220.4 & 92 & $68.24 / 68.51$ & $4.29 / 4.08$ & $19.89 / 19.59$ \\
\hline 2 & $\mathrm{C}_{19} \mathrm{H}_{13} \mathrm{~N}_{3} \mathrm{O}$ (299.33) & 350.7 & 88 & $76.24 / 76.48$ & $4.38 / 4.14$ & $14.04 / 14.31$ \\
\hline 3 & $\mathrm{C}_{20} \mathrm{H}_{15} \mathrm{~N}_{3} \mathrm{O}$ (313.35) & 309.6 & 78 & $76.66 / 76.34$ & 4. $82 / 4.46$ & $13.41 / 13.09$ \\
\hline 4 & $\mathrm{C}_{19} \mathrm{H}_{13} \mathrm{~N}_{3} \mathrm{O}_{2}$ (315.33) & 302.1 & 85 & $72.37 / 72.69$ & $4.16 / 4.39$ & $13.33 / 13.12$ \\
\hline 5 & $\mathrm{C}_{20} \mathrm{H}_{15} \mathrm{~N}_{3} \mathrm{O}_{2}$ (329.35) & 282.2 & 69 & 72.94/72.66 & $4.59 / 4.29$ & $12.76 / 12.44$ \\
\hline 6 & $\mathrm{C}_{20} \mathrm{H}_{15} \mathrm{~N}_{3} \mathrm{O}_{2}$ (329.35) & 245.9 & 84 & 72.94/72.61 & $4.59 / 4.29$ & $12.76 / 12.99$ \\
\hline 7 & $\mathrm{C}_{19} \mathrm{H}_{12} \mathrm{FN}_{3} \mathrm{O}(317.32)$ & 296.5 & 86 & $71.92 / 72.23$ & $3.81 / 3.49$ & $13.24 / 13.56$ \\
\hline 8 & $\mathrm{C}_{21} \mathrm{H}_{15} \mathrm{~N}_{3} \mathrm{O}(325.36)$ & 236.4 & 69 & $77.52 / 77.24$ & $4.65 / 4.41$ & $12.91 / 12.66$ \\
\hline 9 & $\mathrm{C}_{21} \mathrm{H}_{18} \mathrm{~N}_{4} \mathrm{O}$ (342.39) & 287.9 & 77 & 73.67/73.36 & $5.30 / 5.55$ & $16.36 / 16.09$ \\
\hline 10 & $\mathrm{C}_{19} \mathrm{H}_{12} \mathrm{ClN}_{3} \mathrm{O}$ (333.77) & 240.2 & 88 & $68.37 / 68.11$ & $3.62 / 3.33$ & $12.59 / 12.91$ \\
\hline 11 & $\mathrm{C}_{19} \mathrm{H}_{12} \mathrm{ClN}_{3} \mathrm{O}(333.77)$ & 313.7 & 89 & $68.37 / 68.03$ & $3.62 / 3.31$ & $12.59 / 12.88$ \\
\hline 12 & $\mathrm{C}_{20} \mathrm{H}_{13} \mathrm{~N}_{3} \mathrm{O}_{3}(343.34)$ & 276.1 & 79 & $69.96 / 69.68$ & $3.82 / 3.57$ & $12.24 / 12.50$ \\
\hline 13 & $\mathrm{C}_{19} \mathrm{H}_{12} \mathrm{~N}_{4} \mathrm{O}_{3}(344.32)$ & 238.0 & 77 & $66.28 / 66.51$ & $3.51 / 3.22$ & $16.27 / 16.01$ \\
\hline 14 & $\mathrm{C}_{19} \mathrm{H}_{12} \mathrm{~N}_{4} \mathrm{O}_{3}(344.32)$ & 198.7 & 72 & $66.28 / 66.49$ & $3.51 / 3.20$ & $16.27 / 16.00$ \\
\hline 15 & $\mathrm{C}_{20} \mathrm{H}_{15} \mathrm{~N}_{3} \mathrm{O}_{3}(345.35)$ & 304.5 & 66 & $69.56 / 69.77$ & $4.38 / 4.12$ & $12.17 / 12.44$ \\
\hline 16 & $\mathrm{C}_{21} \mathrm{H}_{17} \mathrm{~N}_{3} \mathrm{O}_{3}$ (359.38) & 289.1 & 69 & $70.18 / 70.55$ & $4.77 / 4.33$ & $11.69 / 11.41$ \\
\hline 17 & $\mathrm{C}_{22} \mathrm{H}_{19} \mathrm{~N}_{3} \mathrm{O}_{4}(389.40)$ & 180.7 & 68 & $67.86 / 67.50$ & $4.92 / 4.68$ & $10.79 / 10.45$ \\
\hline 18 & $\mathrm{C}_{19} \mathrm{H}_{11} \mathrm{Cl}_{2} \mathrm{~N}_{3} \mathrm{O}(368.22)$ & 312.1 & 90 & $61.98 / 61.68$ & $3.01 / 3.29$ & $11.41 / 11.72$ \\
\hline 19 & $\mathrm{C}_{23} \mathrm{H}_{20} \mathrm{~N}_{4} \mathrm{O}(368.43)$ & 298.5 & 66 & $74.98 / 74.68$ & $5.47 / 5.12$ & $15.21 / 15.51$ \\
\hline 20 & $\mathrm{C}_{19} \mathrm{H}_{12} \mathrm{BrN}_{3} \mathrm{O}$ (378.22) & 312.6 & 77 & $60.34 / 60.66$ & $3.20 / 3.00$ & $11.11 / 11.39$ \\
\hline 21 & $\mathrm{C}_{19} \mathrm{H}_{12} \mathrm{BrN}_{3} \mathrm{O}$ (378.22) & 332.4 & 78 & $60.34 / 60.01$ & $3.20 / 2.88$ & $11.11 / 10.90$ \\
\hline 22 & $\mathrm{C}_{24} \mathrm{H}_{17} \mathrm{~N}_{3} \mathrm{O}_{2}$ (379.41) & 117.4 & 81 & $75.97 / 75.69$ & $4.52 / 4.21$ & $11.08 / 11.31$ \\
\hline 23 & $\mathrm{C}_{24} \mathrm{H}_{17} \mathrm{~N}_{3} \mathrm{O}_{2}$ (379.41) & 255.5 & 75 & $75.97 / 75.69$ & $4.52 / 4.29$ & $11.08 / 11.31$ \\
\hline 24 & $\mathrm{C}_{17} \mathrm{H}_{11} \mathrm{~N}_{3} \mathrm{OS}(305.35)$ & 241.9 & 89 & $66.87 / 66.51$ & $3.63 / 3.46$ & $13.76 / 13.50$ \\
\hline 25 & $\mathrm{C}_{19} \mathrm{H}_{12} \mathrm{~N}_{2} \mathrm{O}_{3}$ (316.31) & $>350$ & 72 & $72.15 / 72.47$ & $3.82 / 3.99$ & $8.86 / 8.55$ \\
\hline 26 & $\mathrm{C}_{19} \mathrm{H}_{13} \mathrm{~N}_{3} \mathrm{O}_{2}$ (315.33) & 195.6 & 69 & $72.37 / 72.62$ & $4.16 / 4.44$ & $13.33 / 13.00$ \\
\hline 27 & $\mathrm{C}_{23} \mathrm{H}_{14} \mathrm{~N}_{2} \mathrm{O}_{3}(366.37)$ & 160.7 & 68 & $75.40 / 75.71$ & $3.85 / 3.57$ & 7.65/7.98 \\
\hline 28 & $\mathrm{C}_{23} \mathrm{H}_{15} \mathrm{~N}_{3} \mathrm{O}_{2}$ (365.38) & 270.2 & 59 & $75.60 / 75.28$ & $4.14 / 4.39$ & $11.50 / 11.81$ \\
\hline
\end{tabular}

Test compounds were dissolved in DMSO and diluted with saline to the appropriate volume and maintained in RPMI 1640 medium. Different concentrations of each test compound $\left(5,12.5,25\right.$ and $\left.50 \mu \mathrm{mol} \mathrm{L}^{-1}\right)$ were added to the cell monolayer. Triplicate wells were prepared for each individual dose. Monolayer cells were incubated with the compound(s) for $48 \mathrm{~h}$ at $37{ }^{\circ} \mathrm{C}$ and in an atmosphere of $5 \% \mathrm{CO}_{2}$. After $48 \mathrm{~h}$, cells were fixed in situ by gentle addition of $50 \mu \mathrm{L}$ of cold $30 \%(\mathrm{~m} / \mathrm{V})$ TCA (final concentration, $10 \%$ TCA) and incubated for $60 \mathrm{~min}$ at $4{ }^{\circ} \mathrm{C}$. The supernatant was discarded; the plates were washed five times 
with tap water and air dried. Sulforhodamine B solution $(50 \mu \mathrm{L})$ at $0.4 \%(\mathrm{~m} / \mathrm{V})$ in $1 \%$ acetic acid was added to each of the wells and plates were incubated for $20 \mathrm{~min}$ at room temperature. After staining, unbound dye was removed by four washes with $1 \%$ acetic acid and the attached stain was recovered with Tris-EDTA buffer. Color intensity was measured using an ELISA reader (BMG Labtech, Germany). The relation between the surviving fraction and drug concentration was plotted to get the survival curve for the breast cancer cell line (MCF7) after specified time (22). The molar concentration required for $50 \%$ inhibition of cell viability $\left(I C_{50}\right)$ was calculated from the constructed dose-response curve using Prism software (Graphpad, Inc., USA) and compared with the reference drug doxorubicin. Results are given in Table III.

\section{RESULTS AND DISCUSSION}

\section{Chemistry}

The designed target compounds are depicted in Schemes 1 and 2 . In the present investigation, the strategic starting material, 2-cyano- $N$-(quinolin-3-yl) acetamide (1), was

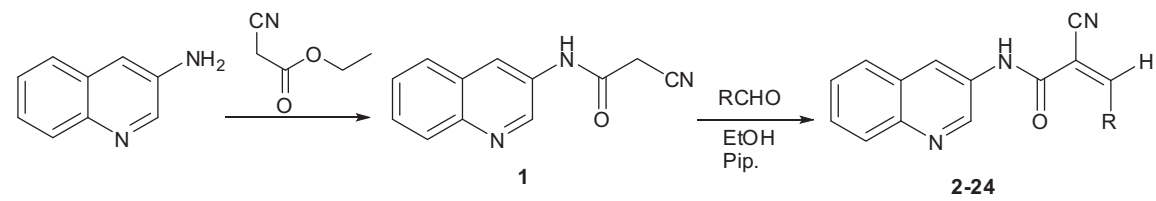

$\mathrm{R}=$

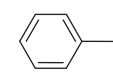

2

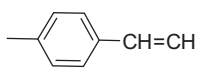

8

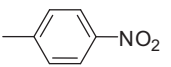

14
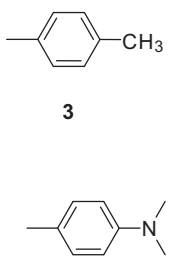

9

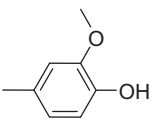

15

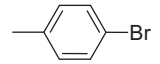

21

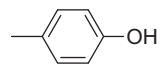

4

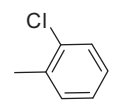

10

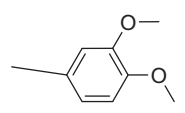

16

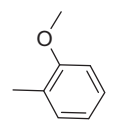

5

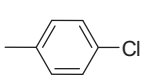

11

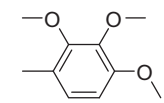

17

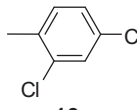

18

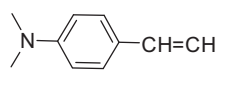

19<smiles>COc1ccc2ccccc2c1C</smiles>

22<smiles>COc1ccc(C)c2ccccc12</smiles>

23

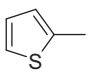

24

Scheme 1 
prepared in good yield via reaction of 3-aminoquinoline with ethyl cyanoacetate under fusion condition. The structure of compound $\mathbf{1}$ was proven on the basis of elemental analysis, IR, ${ }^{1} \mathrm{H}$ NMR and ${ }^{13} \mathrm{C}$ NMR spectral data. In addition, the structure of compound 1 was confirmed by $\mathrm{X}$-ray crystallography (23). IR spectrum showed characteristic bands at $3260 \mathrm{~cm}^{-1}(\mathrm{NH}), 2201 \mathrm{~cm}^{-1}(\mathrm{C} \equiv \mathrm{N}), 1700 \mathrm{~cm}^{-1}(\mathrm{C}=\mathrm{O}), 1617 \mathrm{~cm}^{-1}(\mathrm{C}=\mathrm{N}) .{ }^{1} \mathrm{H}$ NMR spectrum of 1 reveled signals at $4.0 \mathrm{ppm}$ assigned to the $\mathrm{CH}_{2}$ group, 7.5 and $8.6 \mathrm{ppm}$ due to $2 \mathrm{CH}$ quinoline, $10.8 \mathrm{ppm}$ corresponding to the $\mathrm{NH}$ group. ${ }^{13} \mathrm{C}$ NMR spectrum of 1 exhibited a singlet at $161.9 \mathrm{ppm}$ for $(\mathrm{C}=\mathrm{O})$ (Table II). Also, a novel series of potentially active quinoline incorporating cyanoacrylamide moieties (tyrphostin analogs) 2-24 were synthesized through reaction of compound $\mathbf{1}$ with various aldehydes in absolute ethanol containing a catalytic amount of piperidine (Scheme 1). The structures of compounds 2-24 were established on the basis of elemental analyses and spectral data. IR spectra revealed the presence of characteristic bands at $3426-3189 \mathrm{~cm}^{-1}(\mathrm{NH}), 2222-2170 \mathrm{~cm}^{-1}(\mathrm{C} \equiv \mathrm{N}), 1700-1657$ $\mathrm{cm}^{-1}(\mathrm{C}=\mathrm{O}) .{ }^{1} \mathrm{H}$ NMR spectra of compounds $2-24$ revealed the presence of a singlet at 9.0$8.0 \mathrm{ppm}$ assigned to $\mathrm{CH}$ group and 11.1-10.0 ppm for NH group. Furthermore, Perkin reaction (24) was carried out by reaction of salicylaldehyde and/or 2-hydroxy-1naphthaldehyde in acetic anhydride in the presence of sodium acetate to give the corresponding chromene-2-one 25 and benzochromene-2-one 27, while conducting the same reaction in ethanol containing ammonium acetate furnished 2-iminochromene 26 and 2-iminobenzochromene 28 (Scheme 2). The structure of compounds 25-28 was supported of elemental analyses and spectral data. IR spectrum of $\mathbf{2 5}$ revealed bands at $3217 \mathrm{~cm}^{-1}(\mathrm{NH}), 1711,1668 \mathrm{~cm}^{-1}(2 \mathrm{C}=\mathrm{O}) .{ }^{1} \mathrm{H}$ NMR spectrum of 25 showed a singlet at 8.8
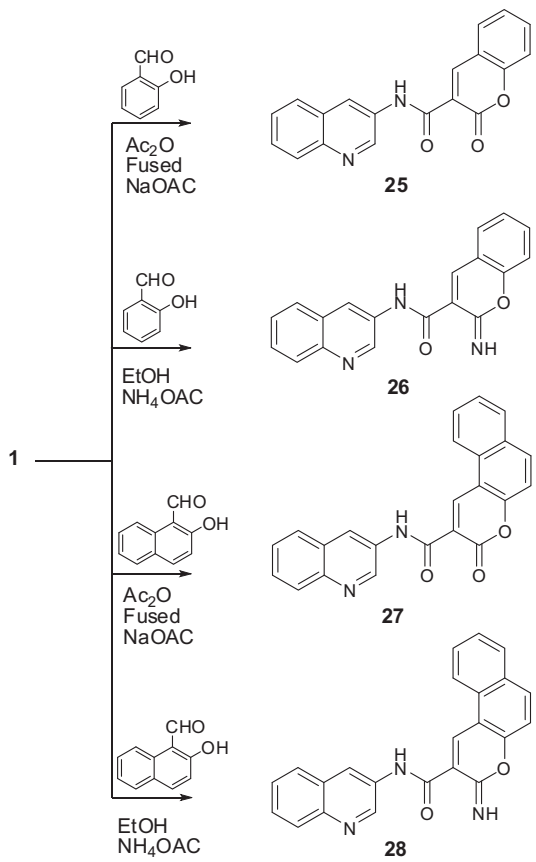<smiles>N=c1oc2ccccc2cc1C(=O)Nc1cnc2ccccc2c1</smiles><smiles>O=C(Nc1cnc2ccccc2c1)c1cc2c(ccc3ccccc32)oc1=O</smiles>

27

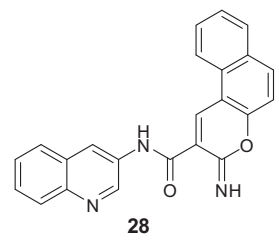

Scheme 2 
ppm, assigned to $\mathrm{CH}$ chromene and $10.4 \mathrm{ppm}$ due to $\mathrm{NH}$ group. ${ }^{13} \mathrm{C}$ NMR spectrum showed signals at 160.8, $164.9 \mathrm{ppm}$ assignable to $2 \mathrm{C}=\mathrm{O}$. IR spectrum of $\mathbf{2 6}$ revealed bands at $3261,3216 \mathrm{~cm}^{-1}(\mathrm{NH}), 1700 \mathrm{~cm}^{-1}(\mathrm{C}=\mathrm{O}) .{ }^{1} \mathrm{H}$ NMR spectrum revealed singlet at $12.6 \mathrm{ppm}$, assigned to the imino group. IR spectrum of 27 exhibited characteristic bands at $3323 \mathrm{~cm}^{-1}$ $(\mathrm{NH}), 1771,1712 \mathrm{~cm}^{-1}(2 \mathrm{C}=\mathrm{O}) .{ }^{1} \mathrm{H}$ NMR spectrum showed a singlet at $8.9 \mathrm{ppm}$, attributed to $\mathrm{CH}$ benzochromene. IR spectrum of 28 revealed bands at $3158,3112 \mathrm{~cm}^{-1}(\mathrm{NH}), 1685 \mathrm{~cm}^{-1}$ $\left((\mathrm{C}=\mathrm{O})\right.$, and ${ }^{1} \mathrm{H}$ NMR spectrum showed a singlet at $12.6 \mathrm{ppm}$ corresponding to the imino group (Table II).

Table II. Spectral characterization of the newly synthesized compounds

\begin{tabular}{|c|c|c|c|}
\hline 宅 & $\left(v_{\max } \mathrm{cm}^{-1}\right)$ & $\begin{array}{l}{ }^{1} \mathrm{H} \text { NMR }\left(\mathrm{DMSO}-d_{6}\right) \\
{ }^{13} \mathrm{C} \text { NMR }\left(\mathrm{DMSO}-d_{6}\right) \\
(\delta, \mathrm{ppm})\end{array}$ & $\begin{array}{l}\text { Mass } \\
(m / z, \%)\end{array}$ \\
\hline 1 & $\begin{array}{l}3260(\mathrm{NH}), 3091(\mathrm{CH} \\
\text { arom.), } 2957,2927(\mathrm{CH} \\
\text { aliph.), } 2201(\mathrm{C} \equiv \mathrm{N}), \\
1700(\mathrm{C}=\mathrm{O}), 1617(\mathrm{C}=\mathrm{N})\end{array}$ & $\begin{array}{l}4.0\left[\mathrm{~s}, 2 \mathrm{H}, \mathrm{CH}_{2}\right], 7.5,8.6[2 \mathrm{~s}, 2 \mathrm{H}, 2 \mathrm{CH} \text { quinoline], } 7.6-7.9 \\
{[\mathrm{m}, 4 \mathrm{H}, \mathrm{Ar}-\mathrm{H}], 10.8\left[\mathrm{~s}, 1 \mathrm{H}, \mathrm{NH}, \mathrm{D}_{2} \mathrm{O}-\text {-exchangeable] }\right.} \\
24.7,115.6,122.6,123.8,125.4,126.3,127.7,128.5,132.0 \text {, } \\
144.1,144.4,161.9\end{array}$ & $\begin{array}{c}211\left[\mathrm{M}^{+}\right](9.4) \\
68(100)\end{array}$ \\
\hline 2 & $\begin{array}{l}3216(\mathrm{NH}), 3100(\mathrm{CH} \\
\text { arom.), 2976, } 2889(\mathrm{CH} \\
\text { aliph.), } 2212(\mathrm{C} \equiv \mathrm{N}), \\
1668(\mathrm{C}=\mathrm{O}), 1586(\mathrm{C}=\mathrm{N})\end{array}$ & $\begin{array}{l}\text { 7.0, } 8.3[2 \mathrm{~s}, 2 \mathrm{H}, 2 \mathrm{CH} \text { quinoline], } 7.1-8.1[\mathrm{~m}, 9 \mathrm{H}, \mathrm{Ar}-\mathrm{H}] \\
8.9[\mathrm{~s}, 1 \mathrm{H}, \mathrm{CH}], 10.1\left[\mathrm{~s}, 1 \mathrm{H}, \mathrm{NH}, \mathrm{D}_{2} \mathrm{O}-\mathrm{exch} \text { angeable] }\right. \\
109.1,113.6,125.4,125.8,126.0,126.8(2), 127.2,127.6, \\
127.9,128.4(2), 128.6,135.5,140.0(2), 144.0,148.0,163.9\end{array}$ & $\begin{array}{c}299\left[\mathrm{M}^{+}\right] \\
(23.6), 78(100)\end{array}$ \\
\hline 3 & $\begin{array}{l}3245(\mathrm{NH}), 3091(\mathrm{CH} \\
\text { arom.), 2926, } 2861(\mathrm{CH} \\
\text { aliph.), } 2200(\mathrm{C} \equiv \mathrm{N}), \\
1659(\mathrm{C}=\mathrm{O}), 1610(\mathrm{C}=\mathrm{N})\end{array}$ & $\begin{array}{l}2.4\left[\mathrm{~s}, 3 \mathrm{H}, \mathrm{CH}_{3}\right], 7.0,8.3[2 \mathrm{~s}, 2 \mathrm{H}, 2 \mathrm{CH} \text { quinoline], } 7.3-8.0 \\
{[\mathrm{m}, 8 \mathrm{H}, \mathrm{Ar}-\mathrm{H}], 8.7[\mathrm{~s}, 1 \mathrm{H}, \mathrm{CH}], 10.8\left[\mathrm{~s}, 1 \mathrm{H}, \mathrm{NH}, \mathrm{D}_{2} \mathrm{O}-\right.} \\
\text { exchangeable] } \\
21.2,105.4,116.2,124.2,125.1,125.8,126.8(2), 127.1, \\
127.5,128.4(2), 129.9,132.0,135.6,137.0,143.4,144.0, \\
151.4,163.9\end{array}$ & $\begin{array}{l}(17.9), \\
00)\end{array}$ \\
\hline 4 & $\begin{array}{l}3320(\mathrm{OH}), 3189(\mathrm{NH}) \\
3065(\mathrm{CH} \text { arom.), } 2972 \\
2836(\mathrm{CH} \text { aliph.), } 2204 \\
(\mathrm{C} \equiv \mathrm{N}), 1687(\mathrm{C}=\mathrm{O}) \\
1607(\mathrm{C}=\mathrm{N})\end{array}$ & $\begin{array}{l}\text { 6.9, } 8.7[2 \mathrm{~s}, 2 \mathrm{H}, 2 \mathrm{CH} \text { quinoline], } 7.0-8.0[\mathrm{~m}, 8 \mathrm{H}, \mathrm{Ar}-\mathrm{H}] \text {, } \\
8.2[\mathrm{~s}, 1 \mathrm{H}, \mathrm{CH}], 9.0\left(\mathrm{~s}, 1 \mathrm{H}, \mathrm{OH}, \mathrm{D}_{2} \mathrm{O}-\text {-exchangeable], } 10.6\right. \\
{\left[\mathrm{s}, 1 \mathrm{H}, \mathrm{NH}, \mathrm{D}_{2} \mathrm{O}-\text {-exchangeable] }\right.} \\
101.4,116.3(2), 116.8,122.7,124.0,127.1,127.5,127.8(2), \\
128.3,128.5,132.2,133.1,144.5,145.4,151.3,161.7,162.1\end{array}$ & $\begin{array}{c}315\left[\mathrm{M}^{+}\right](10.1) \\
78(100)\end{array}$ \\
\hline 5 & $\begin{array}{l}3409(\mathrm{NH}), 3100(\mathrm{CH} \\
\text { arom.), 2966, } 2878(\mathrm{CH} \\
\text { aliph.), } 2217(\mathrm{C} \equiv \mathrm{N}), \\
1682(\mathrm{C}=\mathrm{O}), 1599(\mathrm{C}=\mathrm{N})\end{array}$ & $\begin{array}{l}3.8\left[\mathrm{~s}, 3 \mathrm{H}, \mathrm{OCH}_{3}\right], 7.0,8.6[2 \mathrm{~s}, 2 \mathrm{H}, 2 \mathrm{CH} \text { quinoline], } 7.2- \\
8.0[\mathrm{~m}, 8 \mathrm{H}, \mathrm{Ar}-\mathrm{H}], 8.4[\mathrm{~s}, 1 \mathrm{H}, \mathrm{CH}], 10.3\left[\mathrm{~s}, 1 \mathrm{H}, \mathrm{NH}, \mathrm{D}_{2} \mathrm{O}-\right. \\
\text { exchangeable] } \\
55.0,103.8,113.2,113.9,114.8,120.6,122.4,123.6,124.8, \\
126.6,127.0,128.1,128.5,128.8,134.0,136.7,145.9,154.1, \\
158.0,165.7\end{array}$ & $\begin{array}{c}329\left[\mathrm{M}^{+}\right](29.4) \\
107(100)\end{array}$ \\
\hline 6 & $\begin{array}{l}3324(\mathrm{NH}), 3055(\mathrm{CH} \\
\text { arom.), 2927, } 2861(\mathrm{CH} \\
\text { aliph.), } 2211(\mathrm{C} \equiv \mathrm{N}), \\
1677(\mathrm{C}=\mathrm{O}), 1605(\mathrm{C}=\mathrm{N})\end{array}$ & $\begin{array}{l}3.8\left[\mathrm{~s}, 3 \mathrm{H}, \mathrm{OCH}_{3}\right], 7.1,8.7[2 \mathrm{~s}, 2 \mathrm{H}, 2 \mathrm{CH} \text { quinoline], } 7.2- \\
8.3[\mathrm{~m}, 8 \mathrm{H}, \mathrm{Ar}-\mathrm{H}], 9.0[\mathrm{~s}, 1 \mathrm{H}, \mathrm{CH}], 10.7\left[\mathrm{~s}, 1 \mathrm{H}, \mathrm{NH}, \mathrm{D}_{2} \mathrm{O}-\right. \\
\text { exchangeable]. } \\
55.6,102.9,114.9(2), 116.6,124.1,124.2,127.1,127.5,127.8 \\
(2), 128.3,128.5,132.1,132.7,144.5,145.4,151.0,161.5,162.9\end{array}$ & $\begin{array}{c}329\left[\mathrm{M}^{+}\right] \\
(31.5), 109(100)\end{array}$ \\
\hline
\end{tabular}




\begin{tabular}{|c|c|c|c|}
\hline \multirow[t]{2}{*}{7} & \multirow{2}{*}{$\begin{array}{l}3294(\mathrm{NH}), 3045(\mathrm{CH} \\
\text { arom.), 2916, } 2836(\mathrm{CH} \\
\text { aliph.), } 2196(\mathrm{C} \equiv \mathrm{N}), \\
1691(\mathrm{C}=\mathrm{O}), 1605(\mathrm{C}=\mathrm{N})\end{array}$} & $\begin{array}{l}\text { 7.0, } 8.7[2 \mathrm{~s}, 2 \mathrm{H}, 2 \mathrm{CH} \text { quinoline], } 7.1-8.0[\mathrm{~m}, 8 \mathrm{H}, \mathrm{Ar}-\mathrm{H}], \\
8.2[\mathrm{~s}, 1 \mathrm{H}, \mathrm{CH}], 10.5\left[\mathrm{~s}, 1 \mathrm{H}, \mathrm{NH}, \mathrm{D}_{2} \mathrm{O}-\text { exchangeable }\right]\end{array}$ & \multirow[t]{2}{*}{$\begin{array}{l}317\left[\mathrm{M}^{+}\right](24.9), \\
\quad 93(100)\end{array}$} \\
\hline & & $\begin{array}{l}108.4,114.6(2), 116.7,123.9,124.2,125.3,126.8,127.0(2), \\
127.6,128.5,129.0,135.2,144.1,145.0,153.3,162.3,163.7\end{array}$ & \\
\hline \multirow[t]{2}{*}{8} & \multirow{2}{*}{$\begin{array}{l}3401(\mathrm{NH}), 3046(\mathrm{CH} \\
\text { arom.), 2928, } 2861(\mathrm{CH} \\
\text { aliph.), } 2192(\mathrm{C} \equiv \mathrm{N}), \\
1689(\mathrm{C}=\mathrm{O}), 1599(\mathrm{C}=\mathrm{N})\end{array}$} & $\begin{array}{l}\text { 6.7, } 8.0[2 \mathrm{~d}, 2 \mathrm{H}, \mathrm{PhCH}=\mathrm{CH}-\mathrm{CH}, J=7.6 \mathrm{~Hz}], 6.8-6.9[\mathrm{~m}, \\
1 \mathrm{H}, \mathrm{Ph}-\mathrm{CH}=\mathrm{CH}-\mathrm{CH}], 7.2,8.6[2 \mathrm{~s}, 2 \mathrm{H}, 2 \mathrm{CH} \text { quinoline], 7.3- } \\
7.9[\mathrm{~m}, 9 \mathrm{H}, \mathrm{Ar}-\mathrm{H}], 10.2\left[\mathrm{~s}, 1 \mathrm{H}, \mathrm{NH}, \mathrm{D}_{2} \mathrm{O}-\text { exchangeable] }\right.\end{array}$ & \multirow[t]{2}{*}{$\begin{array}{c}325\left[\mathrm{M}^{+}\right] \\
(51.3), 98(100)\end{array}$} \\
\hline & & $\begin{array}{l}92.4,113.2,123.7,124.5,124.8,125.6,127.0(2), 127.4, \\
127.8,128.6,128.9(2), 130.2,132.4,133.8,136.7,136.9, \\
138.3,144.2,162.7\end{array}$ & \\
\hline \multirow[t]{2}{*}{9} & \multirow{2}{*}{$\begin{array}{l}3408(\mathrm{NH}), 3078(\mathrm{CH} \\
\text { arom.), 2971, } 2836(\mathrm{CH} \\
\text { aliph.), } 2219(\mathrm{C} \equiv \mathrm{N}), \\
1700(\mathrm{C}=\mathrm{O}), 1586(\mathrm{C}=\mathrm{N})\end{array}$} & $\begin{array}{l}3.1\left[\mathrm{~s}, 6 \mathrm{H}, 2 \mathrm{CH}_{3}\right], 6.8,8.6[2 \mathrm{~s}, 2 \mathrm{H}, 2 \mathrm{CH} \text { quinoline }], 7.0-8.1 \\
{[\mathrm{~m}, 8 \mathrm{H}, \mathrm{Ar}-\mathrm{H}], 8.8[\mathrm{~s}, 1 \mathrm{H}, \mathrm{CH}], 10.4\left[\mathrm{~s}, 1 \mathrm{H}, \mathrm{NH}, \mathrm{D}_{2} \mathrm{O}-\mathrm{ex}-\right.} \\
\text { changeable] }\end{array}$ & \multirow[t]{2}{*}{$\begin{array}{c}342\left[\mathrm{M}^{+}\right] \\
(12.6), 78(100)\end{array}$} \\
\hline & & $\begin{array}{l}40.1(2), 108.3,111.7(2), 114.3,123.8,124.3,124.8,125.7,127.0 \\
(2), 127.6,128.5(2), 133.0,140.1,142.4,145.5,156.4,162.7\end{array}$ & \\
\hline
\end{tabular}

$103296(\mathrm{NH}), 3081(\mathrm{CH}$ arom.), 2937, 2861 (CH aliph.), $2170(\mathrm{C} \equiv \mathrm{N})$, $1699(\mathrm{C}=\mathrm{O}), 1598$ $(\mathrm{C}=\mathrm{N}), 749(\mathrm{C}-\mathrm{Cl})$

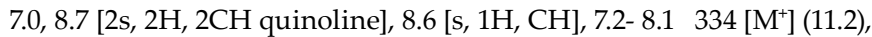
[m, 8H, Ar-H], 10.3 [s, 1H, NH, $\mathrm{D}_{2} \mathrm{O}$-exchangeable] $109(100)$ $107.8,114.4,123.2,124.6,124.9,125.4,127.1,127.8,128.5$, $129.9,129.7,129.9,130.7,131.9,135.7,144.9(2), 155.6,160.1$

$113217(\mathrm{NH}), 3056(\mathrm{CH}$ arom.), 2919, $2836(\mathrm{CH}$ aliph.), $2190(\mathrm{C} \equiv \mathrm{N})$,

6.9, 8.7 [2s, 2H, 2CH quinoline], 7.1- $8.1[\mathrm{~m}, 8 \mathrm{H}, \mathrm{Ar}-\mathrm{H}], 334$ [M+ $\mathrm{M}^{+}(1.9)$, $8.4[\mathrm{~s}, 1 \mathrm{H}, \mathrm{CH}], 10.9\left[\mathrm{~s}, 1 \mathrm{H}, \mathrm{NH}, \mathrm{D}_{2} \mathrm{O}\right.$-exchangeable] $93(100)$ $1676(\mathrm{C}=\mathrm{O}), 1608(\mathrm{C}=\mathrm{N})$, $745(\mathrm{C}-\mathrm{Cl})$ 105.4, 113.6, 122.1, 123.6, 124.8, 127.1, 128.1 (2), 128.4, $128.6(2), 129.1,135.0(2), 136.8,140.6,144.9,151.4,164.7$

$123264(\mathrm{NH}), 3100(\mathrm{CH}$ arom.), 2956, 2861 (CH aliph.), $2186(\mathrm{C} \equiv \mathrm{N})$, $1675(\mathrm{C}=\mathrm{O}), 1581(\mathrm{C}=\mathrm{N})$ $6.2\left[\mathrm{~s}, 2 \mathrm{H}, \mathrm{O}-\mathrm{CH}_{2}-\mathrm{O}\right], 7.1,8.7\left[2 \mathrm{~s}, 2 \mathrm{H}, 2 \mathrm{CH}\right.$ quinoline], $343\left[\mathrm{M}^{+}\right](6.4)$, 7.5- $8.0[\mathrm{~m}, 7 \mathrm{H}, \mathrm{Ar}-\mathrm{H}], 8.6[\mathrm{~s}, 1 \mathrm{H}, \mathrm{CH}], 10.7[\mathrm{~s}, 1 \mathrm{H}, \mathrm{NH}, \quad$ 119(100) $\mathrm{D}_{2} \mathrm{O}$-exchangeable] 102.4, 107.9, 109.1, 116.5 (2), 121.3, 124.1, 125.8, 127.1, $127.5,127.8,128.3,128.5,132.1,138.6,144.5,145.4,148.1$, $151.0,161.4$

$133286(\mathrm{NH}), 3077(\mathrm{CH}$ arom.), 2961, 2881 (CH aliph.), $2210(\mathrm{C} \equiv \mathrm{N})$, $1678(\mathrm{C}=\mathrm{O}), 1612(\mathrm{C}=\mathrm{N})$, $1527,1367\left(\mathrm{NO}_{2}\right)$

$143380(\mathrm{NH}), 3065(\mathrm{CH}$ arom.), 2991, $2884(\mathrm{CH}$ aliph.), $2219(\mathrm{C} \equiv \mathrm{N})$, $1696(\mathrm{C}=\mathrm{O}), 1601(\mathrm{C}=\mathrm{N})$, $1520,1345\left(\mathrm{NO}_{2}\right)$

7.2, $8.7[2 \mathrm{~s}, 2 \mathrm{H}, 2 \mathrm{CH}$ quinoline], 7.3- $8.1[\mathrm{~m}, 8 \mathrm{H}, \mathrm{Ar}-\mathrm{H}]$, $8.3[\mathrm{~s}, 1 \mathrm{H}, \mathrm{CH}], 10.1$ [s, 1H, NH, $\mathrm{D}_{2} \mathrm{O}$-exchangeable] 102.8, 113.6, 119.7, 122.4, 125.1, 126.9 (2), 127.6, 128.4 (2), 129.8, 133.1, 134.6 (2), 141.3, 144.7, 147.2, 152.8, 161.7 7.0, 8.6 [2s, 2H, 2CH quinoline], 7.1- 8.0 [m, 8H, Ar-H], $8.2[\mathrm{~s}, 1 \mathrm{H}, \mathrm{CH}], 10.9\left[\mathrm{~s}, 1 \mathrm{H}, \mathrm{NH}, \mathrm{D}_{2} \mathrm{O}\right.$-exchangeable] 104.3, 116.6, 122.8 (2), 123.7, 126.9 (2), 127.8 (2), 128.0, 128.3, $334\left[\mathrm{M}^{+}\right]$ (10.8), 123 (100) $128.8,136.8,142.1(2), 146.4,147.2,156.7,166.3$. Anal. Calcd for $\mathrm{C}_{19} \mathrm{H}_{12} \mathrm{~N}_{4} \mathrm{O}_{3}(344)$ : $\mathrm{C}, 66.28 ; \mathrm{H}, 3.51 ; \mathrm{N}, 16.27$

$153336(\mathrm{OH}), 3289(\mathrm{NH}), \quad 3.8\left[\mathrm{~s}, 3 \mathrm{H}, \mathrm{OCH}_{3}\right], 7.0,8.8$ [2s, 2H, 2CH quinoline], 7.5- $345\left[\mathrm{M}^{+}\right]$(7.6), 3044 (CH arom.), 2972, 2856 (CH aliph.), 2220 $(\mathrm{C} \equiv \mathrm{N}), 1671(\mathrm{C}=\mathrm{O})$, $1586(\mathrm{C}=\mathrm{N})$ $7.9[\mathrm{~m}, 7 \mathrm{H}, \mathrm{Ar}-\mathrm{H}], 8.4[\mathrm{~s}, 1 \mathrm{H}, \mathrm{CH}], 9.4\left[\mathrm{~s}, 1 \mathrm{H}, \mathrm{OH}, \mathrm{D}_{2} \mathrm{O}-\right.$ exchangeable], $11.1\left[\mathrm{~s}, 1 \mathrm{H}, \mathrm{NH}, \mathrm{D}_{2} \mathrm{O}\right.$-exchangeable] $55.5,100.9,113.3,116.0,116.8,123.0,124.2,126.7,128.0,128.3$, $128.6,129.1,130.8,132.8$ (2), 142.2, 147.8, 152.1, 152.2, 163.8 


\begin{tabular}{|c|c|c|c|}
\hline 16 & $\begin{array}{l}3392(\mathrm{NH}), 3062(\mathrm{CH} \\
\text { arom.), 2931, } 2846(\mathrm{CH} \\
\text { aliph.), } 2210(\mathrm{C} \equiv \mathrm{N}) \\
1683(\mathrm{C}=\mathrm{O}), 1589(\mathrm{C}=\mathrm{N})\end{array}$ & $\begin{array}{l}\text { 3.7, } 3.8\left[2 \mathrm{~s}, 6 \mathrm{H}, 2 \mathrm{OCH}_{3}\right], 7.0,8.6[2 \mathrm{~s}, 2 \mathrm{H}, 2 \mathrm{CH} \text { quinoline], } \\
\text { 7.2- } 7.9[\mathrm{~m}, 7 \mathrm{H}, \mathrm{Ar}-\mathrm{H}], 8.4[\mathrm{~s}, 1 \mathrm{H}, \mathrm{CH}], 10.9[\mathrm{~s}, 1 \mathrm{H}, \mathrm{NH}, \\
\left.\mathrm{D}_{2} \mathrm{O}-\mathrm{exchangeable}\right] \\
56.3(2), 109.3,111.7,112.2(2), 119.2,122.4,123.5,127.1, \\
127.5,128.1,128.7,129.4,134.9,137.3,144.6,148.9,149.0, \\
151.5,165.4\end{array}$ & $\begin{array}{c}359\left[\mathrm{M}^{+}\right] \\
(68.3), 78(100)\end{array}$ \\
\hline 17 & $\begin{array}{l}3314(\mathrm{NH}), 3061(\mathrm{CH} \\
\text { arom.), 2972, } 2855(\mathrm{CH} \\
\text { aliph.), 2215 }(\mathrm{C} \equiv \mathrm{N}) \\
1673(\mathrm{C}=\mathrm{O}), 1599(\mathrm{C}=\mathrm{N})\end{array}$ & $\begin{array}{l}\left.\text { 3.68, 3.72 [2s, } 9 \mathrm{H}, 3 \mathrm{OCH}_{3}\right], 7.1,8.6[2 \mathrm{~s}, 2 \mathrm{H}, 2 \mathrm{CH} \text { quino- } \\
\text { line], 7.2- } 8.0[\mathrm{~m}, 6 \mathrm{H}, \mathrm{Ar}-\mathrm{H}], 8.5[\mathrm{~s}, 1 \mathrm{H}, \mathrm{CH}], 10.2[\mathrm{~s}, 1 \mathrm{H}, \\
\mathrm{NH}, \mathrm{D}_{2} \mathrm{O}-\text { exchangeable] } \\
\text { 55.6, 60.2, 60.8, 105.3, 106.4, 107.6, 114.8, 119.1, 122.6, } \\
\text { 124.4, 125.2, 127.1, 127.8, 128.4, 135.1, 138.5, 138.8, 142.6, } \\
148.7,151.6,152.0,162.6\end{array}$ & $\begin{array}{l}389\left[\mathrm{M}^{+}\right] \\
(36.9), 164 \\
(100)\end{array}$ \\
\hline 18 & $\begin{array}{l}3426(\mathrm{NH}), 3100(\mathrm{CH} \\
\text { arom.), 2970, } 2850(\mathrm{CH} \\
\text { aliph.), } 1688(\mathrm{C}=\mathrm{O}) \\
1629(\mathrm{C}=\mathrm{N}), 747(\mathrm{C}-\mathrm{Cl})\end{array}$ & $\begin{array}{l}\text { 7.2, } 8.7[2 \mathrm{~s}, 2 \mathrm{H}, 2 \mathrm{CH} \text { quinoline], } 7.3-8.1[\mathrm{~m}, 7 \mathrm{H}, \mathrm{Ar}-\mathrm{H}] \\
8.5[\mathrm{~s}, 1 \mathrm{H}, \mathrm{CH}], 10.5\left[\mathrm{~s}, 1 \mathrm{H}, \mathrm{NH}, \mathrm{D}_{2} \mathrm{O}-\text { exchangeable] }\right. \\
105.1,112.6,122.8,125.1,125.8,125.9,126.3,126.9,127.4, \\
128.2,129.7,131.6,133.6(2), 141.2(2), 142.1,152.7,160.2\end{array}$ & $\begin{array}{c}368\left[\mathrm{M}^{+}\right] \\
(111.6), 146 \\
(100)\end{array}$ \\
\hline 19 & $\begin{array}{l}3337(\mathrm{NH}), 3061(\mathrm{CH} \\
\text { arom.), 2971, } 2836(\mathrm{CH} \\
\text { aliph.), 2201 }(\mathrm{C} \equiv \mathrm{N}) \\
1676(\mathrm{C}=\mathrm{O}), 1592(\mathrm{C}=\mathrm{N})\end{array}$ & $\begin{array}{l}3.1\left[\mathrm{~s}, 6 \mathrm{H}, 2 \mathrm{CH}_{3}\right], 6.6,8.0[2 \mathrm{~d}, 2 \mathrm{H},=\mathrm{CH}-\mathrm{CH}=\underline{\mathrm{CH}}+\mathrm{C} ;= \\
7.3 \mathrm{~Hz}], 6.8[\mathrm{~m}, 1 \mathrm{H}, \mathrm{CH}-\mathrm{CH}=\mathrm{CH}-\mathrm{Ph}], 7.0,8.5[2 \mathrm{~s}, 2 \mathrm{H}, \\
2 \mathrm{CH} \text { quinoline], 7.2- } 7.9[\mathrm{~m}, 8 \mathrm{H}, \mathrm{Ar}-\mathrm{H}], 10.8[\mathrm{~s}, 1 \mathrm{H}, \mathrm{NH}, \\
\mathrm{D}_{2} \mathrm{O}-\text { exchangeable] } \\
42.3(2), 94.6,112.8(2), 114.6,123.1,123.8,124.6,124.9, \\
125.7,126.4(2), 127.0,127.6,128.3,129.8,130.9,135.4 \\
138.2,141.6,147.5,162.9\end{array}$ & $\begin{array}{c}368\left[\mathrm{M}^{+}\right](16.4) \\
141(100)\end{array}$ \\
\hline 20 & $\begin{array}{l}3213(\mathrm{NH}), 3067(\mathrm{CH} \\
\text { arom.), 2971, } 2839(\mathrm{CH} \\
\text { aliph.), } 2217(\mathrm{C} \equiv \mathrm{N}) \\
1657(\mathrm{C}=\mathrm{O}), 1607(\mathrm{C}=\mathrm{N})\end{array}$ & $\begin{array}{l}\text { 6.9, } 8.7[2 \mathrm{~s}, 2 \mathrm{H}, 2 \mathrm{CH} \text { quinoline], } 7.0-8.1[\mathrm{~m}, 8 \mathrm{H}, \mathrm{Ar}-\mathrm{H}] \\
8.3[\mathrm{~s}, 1 \mathrm{H}, \mathrm{CH}], 10.0\left[\mathrm{~s}, 1 \mathrm{H}, \mathrm{NH}, \mathrm{D}_{2} \mathrm{O}-\text { exchangeable] }\right. \\
103.7,114.2,121.2,124.4,124.7,125.3,126.9,127.3,127.8, \\
128.1,129.0,129.4,131.0,135.1 \text { (2), 143.0, 144.1, 148.2, } \\
163.5\end{array}$ & $\begin{array}{c}378\left[\mathrm{M}^{+}\right](3.6) \\
155(100)\end{array}$ \\
\hline 21 & $\begin{array}{l}3361(\mathrm{NH}), 3056(\mathrm{CH} \\
\text { arom.), 2956, } 2886(\mathrm{CH} \\
\text { aliph.), } 2222(\mathrm{C} \equiv \mathrm{N}), \\
1670(\mathrm{C}=\mathrm{O}), 1599(\mathrm{C}=\mathrm{N})\end{array}$ & $\begin{array}{l}\text { 7.3, } 8.7[2 \mathrm{~s}, 2 \mathrm{H}, 2 \mathrm{CH} \text { quinoline], 7.4- } 8.1[\mathrm{~m}, 8 \mathrm{H}, \mathrm{Ar}-\mathrm{H}] \text {, } \\
8.5[\mathrm{~s}, 1 \mathrm{H}, \mathrm{CH}], 10.9\left[\mathrm{~s}, 1 \mathrm{H}, \mathrm{NH}, \mathrm{D}_{2} \mathrm{O}-\text { exchangeable] }\right. \\
108.4,118.1,121.1,122.3,125.2,126.6,127.1,127.6,128.0 \\
(2), 128.9,130.9(2), 132.5,135.2,139.7,144.1,157.3,163.7\end{array}$ & $\begin{array}{c}378\left[\mathrm{M}^{+}\right](1.8) \\
81(100)\end{array}$ \\
\hline 22 & $\begin{array}{l}3249(\mathrm{NH}), 3060(\mathrm{CH} \\
\text { arom.), 2935, } 2862(\mathrm{CH} \\
\text { aliph.), } 2193(\mathrm{C} \equiv \mathrm{N}), \\
1669(\mathrm{C}=\mathrm{O}), 1612(\mathrm{C}=\mathrm{N})\end{array}$ & 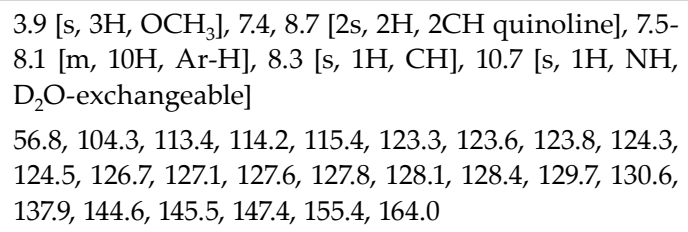 & $\begin{array}{c}379\left[\mathrm{M}^{+}\right](19.7) \\
157(100)\end{array}$ \\
\hline 23 & $\begin{array}{l}3376(\mathrm{NH}), 3100(\mathrm{CH} \\
\text { arom.), 2938, } 2866(\mathrm{CH} \\
\text { aliph.), } 2211(\mathrm{C} \equiv \mathrm{N}) \\
1691(\mathrm{C}=\mathrm{O}), 1601(\mathrm{C}=\mathrm{N})\end{array}$ & $\begin{array}{l}4.1\left[\mathrm{~s}, 3 \mathrm{H}, \mathrm{OCH}_{3}\right], 7.2,8.7[2 \mathrm{~s}, 2 \mathrm{H}, 2 \mathrm{CH} \text { quinoline], } 7.6- \\
8.0[\mathrm{~m}, 10 \mathrm{H}, \mathrm{Ar}-\mathrm{H}], 8.3[\mathrm{~s}, 1 \mathrm{H}, \mathrm{CH}], 10.9[\mathrm{~s}, 1 \mathrm{H}, \mathrm{NH}, \\
\mathrm{D}_{2} \mathrm{O}-\text {-exchangeable] } \\
56.2,104.5,107.2,116.5,121.0,122.3,123.6,124.1,124.7 \\
(2), 126.3,127.1,127.6,127.8,128.2,128.3,128.5,129.4 \\
132.1,144.6,145.5,148.5,158.5,161.4\end{array}$ & $\begin{array}{c}379\left[\mathrm{M}^{+}\right](4.9) \\
149(100)\end{array}$ \\
\hline
\end{tabular}




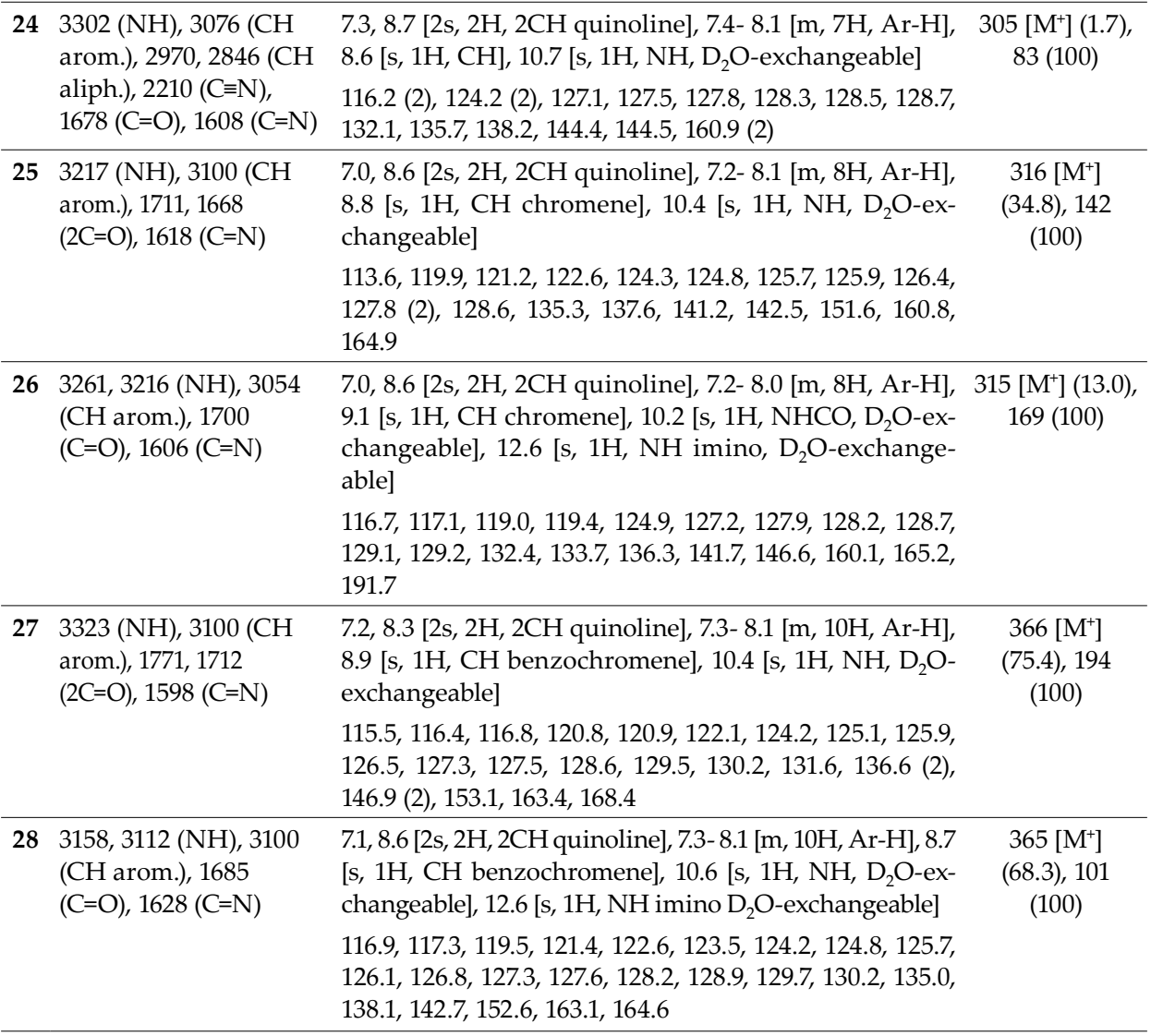

In vitro anti-breast cancer activity

The relationship between the surviving fraction and drug concentration was plotted; the response parameter calculated was $I C_{50}$ value, which corresponds to the compound concentration that causes $50 \%$ inhibition of cellular viability (Table III). From Table III we can see that the tested compounds 15, 27, 7, 19 were found to be more potent than doxorubicin as reference drug $\left(I C_{50}=47.9 \mu \mathrm{mol} \mathrm{L}^{-1}\right)$ on the MCF7 cell line. The most potent compounds in this study are compound 15 bearing 4-hydroxyl,3-methoxyphenyl moiety $\left(I C_{50}\right.$ $\left.=29.8 \mu \mathrm{mol} \mathrm{L}^{-1}\right)$, benzochromene-2-one derivative $27\left(I C_{50}=39.0 \mu \mathrm{mol} \mathrm{L}^{-1}\right)$, 4-fluorophenyl $7\left(I C_{50}=40.0 \mu \mathrm{mol} \mathrm{L}^{-1}\right)$, styrylphenyl derivative $19\left(I C_{50}=40.4 \mu \mathrm{mol} \mathrm{L}^{-1}\right)$. On the other hand, 2,3,4-trimethoxyphenyl $17\left(I C_{50}=49.8 \mu \mathrm{mol} \mathrm{L}^{-1}\right)$, 2-chlorophenyl $10\left(I C_{50}=53.5 \mu \mathrm{mol} \mathrm{L}^{-1}\right)$, pipronyl derivative $12\left(I C_{50}=57.1 \mu \mathrm{mol} \mathrm{L}^{-1}\right)$, 2-methoxy-naphthyl $22\left(I C_{50}\right.$ value $=57.5 \mu \mathrm{mol}$ $\left.\mathrm{L}^{-1}\right)$, 2,4-dichlorophenyl $18\left(I C_{50}\right.$ value $\left.=57.6 \mu \mathrm{mol} \mathrm{L}^{-1}\right)$ and chromene-2-one $25\left(I C_{50}\right.$ value = 
Table III. In vitro cytotoxic activity of the newly synthesized compounds 1-28 against the human breast cancer cell line (MCF7)

\begin{tabular}{|c|c|c|}
\hline Compound & $\begin{array}{c}I C_{50} \\
\left(\mu \mathrm{gL}^{-1}\right)\end{array}$ & $\begin{array}{c}I C_{50} \\
\left.(\mu \mathrm{mol} \mathrm{L})^{-1}\right)\end{array}$ \\
\hline 1 & 22.7 & 107.5 \\
\hline 2 & 23.7 & 79.2 \\
\hline 3 & 23.3 & 74.4 \\
\hline 4 & 29.5 & 93.6 \\
\hline 5 & NA & NA \\
\hline 6 & NA & NA \\
\hline 7 & 12.7 & 40.0 \\
\hline 8 & 20.7 & 63.6 \\
\hline 9 & 33.4 & 97.6 \\
\hline 10 & 17.9 & 53.5 \\
\hline 11 & 40.6 & 121.5 \\
\hline 12 & 19.6 & 57.1 \\
\hline 13 & 21.8 & 65.2 \\
\hline 14 & 21.7 & 63.0 \\
\hline 15 & 10.3 & 29.8 \\
\hline 16 & 23.2 & 64.6 \\
\hline 17 & 19.4 & 49.8 \\
\hline 18 & 21.2 & 57.6 \\
\hline 19 & 14.9 & 40.4 \\
\hline 20 & 41.0 & 108.4 \\
\hline 21 & 42.0 & 111.1 \\
\hline 22 & 21.8 & 57.5 \\
\hline 23 & NA & NA \\
\hline 24 & 41.3 & 135.4 \\
\hline 25 & 18.7 & 59.1 \\
\hline 26 & 22.1 & 70.1 \\
\hline 27 & 14.3 & 39.0 \\
\hline 28 & NA & NA \\
\hline Doxorubicin & 26.3 & 47.9 \\
\hline
\end{tabular}

NA - No activity observed under the adopted experimental conditions.

$59.1 \mu \mathrm{mol} \mathrm{L}{ }^{-1}$ ) were found to be nearly as potent as doxorubicin. In addition, acrylamide derivatives $\mathbf{2}, \mathbf{3}, \mathbf{8}, \mathbf{1 3}, \mathbf{1 4}, \mathbf{1 6}$ and imino-chromene exhibited moderate activity $\left({ } C_{50}=79.2\right.$, $74.4,63.6,65.2,63.0,64.6,70.1 \mu \mathrm{mol} \mathrm{L}^{-1}$, resp). It was found that the least potent compounds were 1, 4, 9, 11, 20, 21 and $24\left(I C_{50}\right.$ values $\left.=107.5,93.6,97.6,121.5,108.4,111.1,135.4 \mu \mathrm{mol} \mathrm{L}^{-1}\right)$. Finally, compounds 5, 6, 23 and 28 showed no activity against the breast cancer cell line 
MCF7. These results attract attention due to the possible use of the newly synthesized acrylamide derivatives carrying 4-hydroxy-3-methoxyphenyl (15), benzochromene-2-one (27) and acrylamide bearing 4-fluorophenyl (7) and styrylphenyl (10) for treatment of breast tumors.

\section{CONCLUSIONS}

In this work, we report the synthesis of a novel series of quinolines bearing a biologically active acrylamide 1-24, chromene-2-one $\mathbf{2 5}$, chromene-2-imino $\mathbf{2 6}$, benzochromene-2-one 27 and benzochromene-2-imino 28 moieties through simple and convenient routes. The new derivatives were evaluated for their anticancer activity against a human tumor breast cancer cell line (MCF7). It was found that the most potent compounds in this study were the corresponding 2-cyano-3-(4-hydroxy-3-methoxyphenyl)-N-(quinolin-3-yl) acrylamide (15), 3-oxo-N-(quinolin-3-yl)-3H-benzol[f]chromene-2-carboxamide (27), 2-cyano-3(4-fluorophenyl-N-(quinolin-3-yl) acrylamide (7), 2-cyano-5-(4-(dimethylamino) phenyl)$\mathrm{N}$-(quinolin-3-yl) penta-2,4-dienamide (19) compared to doxorubicin as a positive control.

Acknowledgments. - The authors extend their sincere appreciation to the Deanship of Scientific Research at King Saud University for its funding of this research through the Research Group Project No. RGP-VPP- 302.

\section{REFERENCES}

1. S. Levy and S. J. Azoulay, Story about the origin of quinquina and quinidine, J. Cardiovasc. Electrophysiol. 5 (1994) 635-636; DOI: 10.1111/j.1540-8167.1994.tb01304.x.

2. K. F. Wenckcbach, Cinchona derivatives in the treatment of heart disorders, JAMA 81 (1923) 472 474; DOI: 10.1001/jama.1923.02650060042012.

3. A. B. Adnan, A. E. Ola, A. Elsayed and J. I. Young Park, Tetrazolo[1,5-a]quinoline as a potential promising new scaffold for the synthesis of novel anti-inflammatory and antibacterial agents, Eur. J. Med. Chem. 39 (2004) 249-255; DOI: 10.1016/j. ejmech.2003.12.-005.

4. E. Sumesh, V. A. Airody and N. S. Suchetha, Synthesis and antimicrobial activities of novel quinoline derivatives carrying 1, 2, 4-triazole moiety, Eur. J. Med. Chem. 44 (11) (2009) 4637-4647; DOI: 10.1016/j.ejmech.2009.06.031.

5. M. Zhongze, H. Yoshio, N. Taro and C. Yingjie, Novel quinazoline-quinoline alkaloids with cytotoxic and DNA topoisomerase II inhibitory activities, Bioorg. Med. Chem. Lett. 14 (2004) 1193-1196; DOI: 10.1016/j.bmcl.2003.12.048.

6. E. Sumesh, V. A. Airody and R. A. Kumar, New 1,3-oxazolo [4,5-c] quinoline derivatives: Synthesis and evaluation of antibacterial and antituberculosis properties, Eur. J. Med. Chem. 45 (2010) 957-966; DOI: 10.1016/j.ejmech.2009.11.036.

7. M. Hranjec, M. Kralj, I. Piantanida, M. Sedic, L. Šuman, K. Pavelic and G. Karminski-Zamola, Novel cyano- and amidino-substituted derivatives of styryl-2-benzimidazoles and benzimidazo[1,2- $a$ ] quinolines. Synthesis, photochemical synthesis, DNA binding, and antitumor evaluation, part 3, J. Med. Chem. 50 (2007) 5696-5711; DOI: 10.1021/jm070876h.

8. S. Madapa, Z. Tusi and S. Batra, Advances in the syntheses of quinoline and quinoline-annulated ring systems, Curr. Org. Chem. 12 (2008) 1116-1183; DOI: 10.2174/138527208785740300.

9. P. Muthumani, S. Venkataraman, R. Meera, N. Govind, N. Chidambaranathan P. Devi and B. Kameswari, Synthesis and biological screening of some novel quinoline derivatives, Pharma Chem. 2 (2010) 385-396. 
10. J. Borran, A. Scozzafava, L. Menabuoni, F. Mincione, F. Briganti, G. Mincione and C. T. Supuran, Carbonic anhydrase inhibitors: synthesis of water-soluble, topically effective intraocular pressure lowering aromatic/heterocyclic sulfonamides containing 8-quinoline-sulfonyl moieties: is the tail more important than the ring?, Bioorg. Med. Chem. 7 (1999) 2397-2406; DOI: 10.1016/S09680896(99)00190-X.

11. V. K. Aqrawal, S. Bano, C. T. Supuran and P. V. Khadikar, QSAR study on carbonic anhydrase inhibitors: aromatic/heterocyclic sulfonamides containing 8-quinoline-sulfonyl moieties, with topical activity as antiglaucoma agents, Eur. J. Med. Chem. 39 (2004) 593-600; DOI: 10.1016/j.ejmech.2004.03.002.

12. W. Peczynska-Czoch, F. Pognan, L. Kaczmarek and J. Boratynski, Synthesis and structure-activity relationship of methyl-substituted indolo [2,3- $b$ ]quinolines: Novel cytotoxic, DNA topoisomerase II inhibitors, J. Med. Chem. 37 (1994) 3503-3510; DOI: 10.1021/jm-00047a008.

13. M. S. Al-Dosari, M. M. Ghorab, M. S. Alsaid, Y. M. Nissan and A. B. Ahmed, Synthesis and anticancer activity of some novel trifluoromethylquinolines carrying a biologically active benzenesulfonamide moiety, Eur. J. Med. Chem. 69 (2013) 373-383; DOI: 10.1016/j.ejmech.-2013.08.048.

14. M. M. Ghorab, Z. H. Ismail, M. Abdalla and A. A. Radwan, Synthesis, antimicrobial evaluation and molecular modelling of novel sulfonamides carrying a biologically active quinazoline nucleus, Arch. Pharm. Res. 36 (2013) 660-670; DOI: 10.1007/s12272-013-00946.

15. M. M. Ghorab, Z. H. Ismail, A. A. Radwan and M. Abdalla, Synthesis and pharmacophore modeling of novel quinazolines bearing a biologically active sulfonamide moiety, Acta. Pharm. 63 (2013) 1-18; DOI: 10.2478/acph-2013-0006.

16. M. S. Al-Dosari, M. M. Ghorab, M. S. Alsaid and Y. M. Nissan, Discovering some novel 7-chloroquinolines carrying a biologically active benzenesulfonamide moiety as a new class of anticancer agents, Chem. Pharm. Bull. 61 (2013) 50-58; DOI: 10.10-02/chin.201328146.

17. D. A. Abou El Ella, M. M. Ghorab, H. I. Heiba and A. M. Soliman, Synthesis of some new thiazolopyrane and thiazolopyranopyrimidine derivatives bearing a sulfonamide moiety for evaluation as anticancer and radiosensitizing agents, Med. Chem. Res. 21 (2012) 2395-2407; DOI: 10.1007/s00044-011-9751-9.

18. M. M. Ghorab, M. S. Alsaid and Y. M. Nissan, Dapson in heterocyclic chemistry, part VI: Synthesis and molecular docking of some novel sulfonebiscompounds of expected anticancer activity, Arzneimittel-forsch. 62 (2012) 497-507; DOI: 10.1055/s-0032-1323660.

19. M. M. Ghorab, M. A. Shaaban, H. M. Refaat, H. I. Heiba and S. S. Ibrahim, Anticancer and radiosensitizing evaluation of some new pyranothiazole-Schiff bases bearing the biologically active sulfonamide moiety, Eur. J. Med. Chem. 53 (2012) 403-407; DOI: 10.1016/j.-ejmech.2012.04.009.

20. M. M. Ghorab, F. A. Ragab, H. I. Heiba, Y. M. Nissan and W. M. Ghorab, Novel brominated quinoline and pyrimidoquinoline derivatives as potential cytotoxic agents with synergistic effects of gamma-radiation, Arch. Pharm. Res. 8 (2012) 1335-1346; DOI: 10.1007/s-12272-012-0803-6.

21. M. M. Ghorab, M. S. Alsaid and Y. M. Nissan, Dapson in heterocyclic chemistry, part V: Synthesis, molecular docking and anticancer activity of some novel sulfonylbis-compounds carrying biologically active dihydropyridine, dihydroisoquinoline, 1,3-dithiolan, 1,3-dithian, acrylamide, pyrazole, pyrazolopyrimidine and benzochromene moieties, Chem. Pharm. Bull. 60 (2012) 10191028; DOI: org/10.-1248/cpb.c12-00292.

22. P. Skehan, R. Storeng, D. Scudiero, A. Monks, J. McMahon, D. Vistica, J. T. Warren, H. Bokesch, S. Kenney and M. R. Boyd, New colorimetric cytotoxicity assay for anticancer-drug screening, J. Natl. Cancer. Inst. 82 (1990) 1107-1112; DOI: 10.1093/jnci/-82.13.1107.

23. M. M. Ghorab, M. S. Alsaid, H. A. Ghabour and H. K. Fun, Synthesis, crystal structure and antitumor activity of novel 2-cyano-N-(quinolin-3-yl) acetamide, Asian. J. Chem. 26 (2014) 7389-7392; DOI: 10.14233/ajchem.17050.

24. Y. Ogata and M. Tsuchida, Kinetics and mechanism of the Perkin reaction, J. Org. Chem. 24 (1959) 78-83; DOI: 10.1021/jo01083a023. 